\title{
Passano i tempi, cambiano i luoghi, mutano le norme. Sostanze psicotrope dall'antichità ai giorni nostri: un uso tra il voluttuario, il sanitario ed il sacro-rituale
}

\author{
Massimo Corti*, Guido Ferreri Ibbadu^
}

\begin{abstract}
SUMMARY
- In this article we are presenting a synthesis of historical, cultural and legal aspects of psychotropic substance, legal and illegal.

We have tried to correlate these legal aspects with the two principal degrees of harm of potential misuse, the classical one of NIDA and that more recent of David Nutt (2007).

It seems that in the conclusions, the choices preferred by the legislators, both in a non direct cause (effect cause) with public health aspects o individual, but with regard to a multitude of other factors such as the right cultural aspects of this population, religion aspects, economic aspects but also ideological aspects and/or ethical moral.
\end{abstract}

Keywords: History of Substance abuse, Laws and regulations, Assessment of harm.

Parole chiave: Storia delle sostanze d'abuso, Normativa, Valutazione del danno.

Una sostanza stupefacente è una sostanza, naturale o sintetica, in grado di alterare, una volta introdotta nell'organismo, le funzioni psichiche e le condizioni fisiche di un individuo determinando in diverso grado fenomeni di dipendenza, di tolleranza e di assuefazione.

Le sostanze stupefacenti possono essere classificate in base a diversi criteri:

- in base agli aspetti normativi (legali o illegali);

- in base alla sintomatologia che inducono (eccitanti, sedative, allucinogene, sedative ipnotiche, inebrianti);

- in base alla struttura del principio attivo (derivati morfinici, amfetaminici, cocaina, THC, alcol ecc.);

- in base alla provenienza (naturali o sintetiche);

- in base alla tossicità (scala di tossicità).

Per indicare le sostanze stupefacenti si utilizza, frequentemente e nel linguaggio comune, il termine "droga".

Questo termine si presta a diverse accezioni, dopo aver escluso quello di sostanza vegetale aromatica che serve a dar sapore ai cibi che lega il concetto di droga a quello di oggetto proveniente da culture estranee e misteriose (spezia), l'attenzione non può che andare a quelle sostanze che, in virtù dei loro effetti farmacologici sul sistema nervoso centrale, e in particolare sullo stato di coscienza, sono fatte oggetto di uso non terapeutico, principalmente voluttuario.

Ma questa definizione spesso non è sufficiente a caratterizzarne il significato.

\footnotetext{
* Medico Responsabile Ser.D. Treviglio.

${ }^{\wedge}$ Medico Ser.D. Martinengo.

ASST Bergamo Ovest.
}

Forse immediatamente porremo l'attenzione sulle caratteristiche dell'azione che questa sostanza è in grado di esplicare e quindi si parlerà di una sostanza psicoattiva capace di alterare l'equilibrio psicofisico della persona (sostanza stupefacente), ma in questo caso il caffè è una droga?

Quindi subito dopo inevitabilmente si porrà I'accento su aspetti di tipo normativi arrivando probabilmente al concetto di "droga" come sostanza psicoattiva illegale.

Differenziando pertanto quest'ultima da tutte quelle sostanze psicoattive utilizzate in ambito farmacologico o ricreazionale e che, con alcune limitazioni, sono però legali nell'uso, acquisto o cessione.

Di fatto è la normativa a caratterizzare una droga da una sostanza che droga "non è"

\section{Aspetti storico-culturali e normativi}

Afrodisiaci: si conosce nell'antica Grecia I'utilizzo di Santoreggia (rosmarino selvatico) chiamato propriamente "erba del satiro" per indicarne appunto le qualità afrodisiache; tale erba sembra fosse utilizzata come "facilitatore" per i riti orgiastici; sempre come stimolante sessuale nell'antica Cina venivano utilizzati decotti di Ginseng mentre gli indigeni dell'Amazzonia la corteccia dell'albero "Muira Puama" e in Messico la Damiana, foglie di un piccolo cespuglio.

Normativa: il "muira Puama" e la Damiana sono tutt'ora commercializzate come tonici e stimolanti a livello generale oltre che per la cura della libido e disfunzioni erettili (www.fitoterapiablog.it/virile-al-top-con-la-damiana-e-la-muira-puama). 
Oppio: è noto nei Sumeri l'utilizzo di oppio già nel 4000 a.C. Hul gil, I'ideogramma con cui indicavano il papavero da oppio, stava per pianta della gioia.

Nell'antichità classica vi era utilizzo diffuso di oppio in tutto l'area del mediterraneo (antica Grecia, Roma, Egitto) soprattutto in ambito medico.

Famoso era un farmaco, il "Galenos" considerato una vera panacea in cui l'oppio era diluito con alcol.

Stando agli scritti di Galeno si ritiene che l'imperatore Marco Aurelio fosse diventato dipendente da oppio.

Un mito raccontava come Demetra, la dea della terra e sorella di Zeus, usasse il papavero per alleviare il dolore provocatole dal rapimento della figlia Persefone.

Per questa ragione, esso veniva usato nel culto ufficiale di tale divinità e il papavero veniva collocato immancabilmente tra le spighe di grano che Demetra tiene in mano nelle raffigurazioni. Gli arabi utilizzavano l'oppio come principio curativo importantissimo.

L'alchimista/medico Paracelso nel XVI secolo diffuse in Europa un preparato a base d'oppio e alcol oltre che zucchero cannella e chiodi di garofano destinato a diventare molto popolare: il laudano o tintura di oppio.

Importante uso di questo si fece nella guerra di Secessione americana per alleviare i dolori dei feriti. Nel 1898, infine, la Bayer lancia sul mercato un nuovo prodotto, uno sciroppo contro il dolore e la tosse contenente diacetilmorfina (l'eroina).

Normativa: nel '700 in Cina l'uso si diffuse nella popolazione e ne fu vietata l'importazione dall'India, importazione gestita dalla società inglese Compagnia delle Indie, e questo causò la "Guerra dell'oppio".

La Cina fu quindi militarmente costretta ad accettare tale importazione e uso che continuò sino al 1906 quando fu nuovamente dichiarata illegale anche se nel 1946 all'avvento del maoismo gli oppiomani furono stimati in ancora 40 milioni.

Utilizzo molto diffuso in Europa nel l'800 tra scrittori e poeti, la letteratura è piena di oppiomani, dall'autobiografico "Confessioni di un oppiomane" di Thomas De Quincey che tra i personaggi da Sherlock Holmes fino ad Anna Karenina con il laudano!

L'oppio e i suoi derivati nel 1912 furono messi al bando in occidente, insieme alla cocaina, dalla convenzione dell'Aja (primo trattato internazionale di controllo dei traffici di droga).

Ultimo stato europeo a proibire l'eroina è stato il Portogallo nel 1962 (www.prassi.cnr.it/prassi/attilnternazionali.html?id=318).

Cannabis: in India la "cannabis indica" era ritenuta di origine divina, in quanto derivava dalla metamorfosi dei peli della schiena di Visnù.

Bevanda preferita da Indra, re degli dei, era coltivata dai bramini negli orti dei templi e serviva alla preparazione di un infuso chiamato bhang (o in periodi precedenti conosciuto come "soma"), che assunto in determinate occasioni rituali favoriva l'unione con la divinità.

Uso molto diffusa nel modo islamico (hashish significa erba) e descritta da Marco Polo nel Milione. Nel mito si raccontava di come l'imam Hasan, a capo della città fortezza di Alamut si servisse dell'hashish per arruolare dei giovani e renderli privi di volontà e da lui assolutamente dipendenti in modo tale da spingerli nelle imprese più pericolose, non escluso l'omicidio.

Anche nel mondo europeo antico non era sconosciuta in quanto si ritiene venisse usata nei culti Germanici di Freya, Dea delI'amore e della fertilità, era molto probabilmente anche cognita ai celti. Il filosofo greco Democrito (460 a.C.), che parla di una bevanda a base di vino, canapa e mirra, usata per produrre visioni; come pure fosse hashish la bevanda indicata nell'Odissea con il nome di "nephente" (dalle parola "phente", ansia, preceduta da negativo - ora diremmo "ansiolitico").

Nel medioevo il sacerdote inglese Robert Burton ne descriveva I'efficacia nella terapia della depressione (The Anatomy of melancholy).

Nell'800 l'Europa riscopriva I'hashish grazie alle conquiste di Napoleone in oriente.

Sebbene durante la campagna d'Egitto (1798), Napoleone emanasse un decreto che vietava ai soldati francesi di "bere il forte liquore fatto dai musulmani con un'erba detta hashish e fumare le foglie della cannabis" al ritorno della spedizione in Europa I'uso dell'hascisc prese a diffondersi, soprattutto a Parigi, come una moda.

Dal 1842, descritto dal prof O'Shaughnessy come ottimo farmaco analgesico e rilassante, inizio a diffondersi tra medici inglesi e anche il medico della Regina Vittoria usava con la sovrana preparazione a base di cannabis.

Si ritiene che da prima del mille a.C. e fino alla fine dell' 800 la cannabis costituisse la coltivazione piu' diffusa sul pianeta e che alla fine dell' 800 tra l' 80 e il $90 \%$ della carta e gran parte dei tessuti fossero fatti con canapa.

Nel 1937 DuPont brevettò la fibra di nylon e la carta veniva in questo periodo prodotta con il legno senza più necessità di canapa.

Normativa: il primo paese a proibire la coltivazione della cannabis fu l'Egitto nel 1879 .

A partire dal 1910 comincio nella zona di New Orleans una intensa campagna stampa contro la marijuana (utilizzata principalmente come sostanza ricreazionale da messicani, musicisti jazz e gente di colore) contro il "pericolo marijuana" e arrivando nel 1915 nella messa al bando in alcune zone del Texas e in California.

Il Marijuana Tax Act del 1937 è la legge che diede il via al proibizionismo nei confronti dell'uso e coltivazione della cannabis negli Stati Uniti e che in seguito si estese a numerosi altri paesi del mondo.

Anche in Italia negli anni trenta il regime fascista dichiarò I'hashish, pochissimo conosciuto in Italia, "nemico della razza" e "droga dei negri"

Attualmente permangono significative differenze normative nei vari Paesi:

Argentina: dopo pronunciamento della Corte Suprema è stato depenalizzato il consumo personale in luogo privato.

Germania: la legge sugli stupefacenti in Germania rimane piuttosto vaga e soggetta ad interpretazioni, uso personale tollerato, possesso di modiche quantità (generalmente entro i 10 grammi, 2-3 grammi in Baviera) non perseguito.

Giamaica: formalmente illegale, ma in realtà ampiamente tollerata, dato che è consumata dal $70 \%$ della popolazione.

Italia: illegale detenzione, coltivazione, vendita; inserita nelle stesse tabelle di eroina e cocaina. Sanzioni amministrative per I'uso personale.

Paesi Bassi: formalmente illegale ma il governo ne permette l'uso secondo termini ben precisi, se maggiorenne in quantità inferiore a 5 grammi al giorno nei coffee-shop autorizzati.

L'importazione e l'esportazione di cannabis è sanzionata con pene fino a quattro anni di carcere. Dal 2003 le farmacie olandesi consentono l'acquisto dietro prescrizione medica di cannabis terapeutica oltre che a farmaci derivati dalla cannabis. 
Svizzera: illegali possesso e coltivazione, le autorità sanitarie elvetiche hanno approvato l'uso di farmaco derivato da cannabis.

Regno Unito: illegale, la cannabis è considerata una sostanza stupefacente di classe B (rischio moderato).

Solitamente nelle prime due occasione in cui si viene trovati in possesso di derivati della cannabis c'è solo una segnalazione alla polizia e il sequestro della sostanza.

Francia: I'uso ricreativo, la vendita e la coltivazione sono punite. Nel gennaio 2014 è stato approvato l'uso di farmaco derivato da cannabis (sclerosi multipla, gravi spasmi muscolari resistenti ad altre terapie).

Spagna: in Spagna la vendita e il consumo in pubblico sono illegali e punite, mentre è tollerato l'uso domestico, la coltivazione fino a tre/cinque piante in luoghi privati.

Normalmente viene considerato spaccio quantitativi di cannabis superiori a 40 grammi

Brasile: illegale ma depenalizzato il possesso inferiore a 20 gr. e il consumo.

La normativa non prevede più il carcere per il possesso ai fini del consumo personale di qualsiasi tipo di droga, ma misure alternative alla detenzione (segnalazione, servizi comunitari e educazione sugli effetti della droga).

Paraguay: il Paraguay nel 1988 ha depenalizzato il possesso - sia a fine medici che ricreativi - fino a 10 grammi di cannabis e 2 grammi di oppiacei.

Stati Uniti: dal 2012, in Colorado, Oregon, Alaska e nello Stato di Washington è passata un'iniziativa legislativa per consentire la coltivazione, la vendita e il consumo di cannabis in luogo privato anche per fini ricreativi.

Nel novembre 2016 la California e il Massachusetts hanno approvato l'uso ricreativo della marijuana (Preposition 64).

La misura approvata in California prevede che lo stato consideri la marijuana come l'alcool, varando quindi misure per controllare e tassare la produzione legale della droga leggera.

Secondo la misura, i maggiori di 21 anni potranno possedere 28 grammi di marijuana per uso personale e piantare a casa fino a sei piante.

Lo Stato poi concederà licenze per la vendita della droga leggera, applicando un'accisa del 15\%. Nel 1996 la California divenne il primo Stato americano a legalizzare l'uso terapeutico.

Ad oggi sono 22 gli Stati e il Distretto di Columbia, ad aver approvato regolamenti e permesso di depenalizzare o legalizzare la produzione e l'uso della cannabis a fini terapeutici sebbene la legge federale sugli stupefacenti non riconosca alcun uso medico legale per i derivati della cannabis.

Portogallo: dal 2001 il Portogallo è diventato il primo Paese al mondo a depenalizzare il consumo personale di tutte le droghe. Coloro trovati in possesso di un quantitativo non superiore alla dose per dieci giorni (25 grammi di marijuana, 2 grammi di cocaina, 1 grammo di eroina, 1 grammo di MDMA) possono subire però una serie di provvedimenti amministrativi.

Non è permesso l'uso terapeutico della cannabis.

Belgio: dal gennaio 2005, il consumo all'interno di luoghi priva$\mathrm{ti}$, il possesso fino a tre grammi e la coltivazione di una pianta di marijuana sebbene rimangano illegali, sono tuttavia tollerati.

Uruguay: nel luglio 2013 la Camera dei Rappresentanti di Montevideo ha approvato una legge che regola la produzione, la vendita e il consumo di cannabis. I'Uruguay pertanto ha come primo Stato a legalizzare l'uso di cannabis per scopi medici, industriali e ricreativi.
Coca: nelle Ande centrali, si ritiene che l'uomo abbia cominciato a masticare le foglie di coca in epoche precedenti al 2500 a.C. Dato il carattere sacrale della coca, la consuetudine e le leggi incaiche ne limitavano l'uso all'aristocrazia imperiale e alla casta sacerdotale.

Dopo il 1500 d.c. con la caduta dell'impero incaico gli indios peruviani cominciavano a fare libero uso della coca.

Gli spagnoli usarono dunque la coca come compenso per il massacrante lavoro effettuato dai nativi nelle miniere e nelle piantagioni degli Incas.

La cocaina fu isolata nella seconda metà dell'800 e in poco tempo l'uso di questa si diffuse tantissimo, Freud usava la cocaina per combattere gli stati depressivi.

La usò ampiamente su di se e sui suoi pazienti e scrisse un saggio intitolato "Sulla cocaina" e pubblicato nel 1884.

Angelo Mariani, un farmacista corso, mise a punto nel 1863 una bevanda a base di vino Bordeaux ed estratti di foglie di coca, chiamato appunto "Vin Mariani".

Questa bevanda diventò straordinariamente popolare tanto che papa Leone XIII concesse a Mariani una medaglia d'oro in segno di riconoscenza.

La stessa coca-cola è stata inizialmente una variante del vin Mariani ideata nel 1886 da J.S.Pemberton a causa del proibizionismo che rendeva impossibile continuare ad utilizzare l'alcol nella preparazione.

Normativa: la cocaina nel 1912 venne messa al bando insieme agli oppiacei con la Convenzione dell'Aja

Allucinogeni: l'utilizzo del "fungo magico" o "carne di Dio" contenente psilocibina era diffuso tra gli sciamani messicani; gli aztechi, invece, utilizzavano principalmente la mescalina e ritenevano sacro il cactus peyote da cui si ricava.

Entrambi gli alcaloidi causano effetti simili all'LSD.

L'esperienza di trascendenza e di illuminazione che questa sostanze causano diventavano parte integrante dei riti religiosi sciamanici. LSD (dietilammide-25 dell'acido lisergico) viene sintetizzato per la prima volta nel 1938 nei Laboratori Sandoz di Basilea da Albert Hofmann, è basato sull'acido lisergico, che si trova nell'ergot, un fungo parassita della segale..., non causa "allucinazioni" in senso proprio, ma amplificazioni dei sensi e distorsioni della percezione della realtà causa con possibile apparsa di disturbi psicotici.

Normativa: LSD Inizialmente sperimentato come farmaco psichiatrico fu successivamente bandito ed è una sostanza illegale. La mescalina diverrà illegale negli Stati Uniti nel 1970, atto che sarà seguito a ruota dalle altre nazioni.

Tuttavia, all'inizio degli anni novanta, la Native American Church verrà autorizzata, secondo le leggi della libertà religiosa, a praticare l'utilizzo rituale del peyote, ritenuto un sacramento. In Italia un decreto ministeriale del 1967 ha previsto l'inserimento di psilocibina e psilocina tra le sostanze illecite, specificando che sono vietati anche i "Funghi del genere strofaria, conocybe e psilocybe".

La psilocybe semilanceata per altro è presente nei boschi anche in Italia.

Amfetamine: I'Efedra sinica è un arbusto che veniva usato da millenni in Cina come stimolante in grado di curare vari problemi ed in particolare l'asma.

Nel 1924 venne sintetizzata la prima amfetamina, l'efedrina, che cominciò ad essere venduta col nome di benzedrina senza prescrizione e con inalatore per la cura dell'asma. 
Normativa: l'efedrina è considerata come sostanza dopante e inclusa nell'elenco statunitense delle "smart drugs", commercializzate negli "smart shops": esercizi commerciali che rivendono sostanze naturali.

Molto utilizzata nella seconda guerra mondiale negli anni 50 venne regolamentata la produzione e vendita per il diffusissimo uso nella popolazione.

Ectasy (MDMA): sintetizzata nel 1912 dai laboratori Merk, negli anni '50 è stata testata come "siero della verità" dai servizi segreti militari americani, dagli anni '60 sino agli anni '70 del '900 è stata utilizzata da psicoterapeuti americani come "strumento psicoterapeutico", ovvero sfruttando le caratteristiche entactogene della sostanza si "aiutava" il paziente nella analisi del profondo e nella relazione con lo psicoterapeuta; in questo periodo è stata anche definita "penicillina per l'anima".

Normativa: nel 1985 in seguito ad un aumento generalizzato del consumo veniva messa al bando negli Stati Uniti e in Europa.

Salvia Divinorum: (salvia dei veggenti) deve il suo nome all'impiego che tradizionalmente ne viene fatto dagli indigeni Mazatechi, che masticandone le foglie o attraverso infusi, si servono delle proprietà psichedeliche di questa pianta soprattutto nella divinazione.

Normativa: recentemente ancora sostanza di libera vendita negli smart shop italiani, dal 2005 è stata aggiunta alla lista sostanze vietate (Gazzetta Ufficiale n. 54 del 7 marzo 2005) e quindi resa illegale.

Khat (Quat): per Khat si intendono le foglie e i germogli della Chata, contiene un alcaloide psicoattivo simile all'efedrina a spiccato effetto psicotropo, euforizzante con capacità di reprimere gli stimoli di fame e fatica; ha anche un notevole effetto analgesico.

Secondo una leggenda etiope il quat sarebbe nato da una goccia d'elisir di vita eterna.

Normativa: considerato una droga illegale ed è sottoposto a misure di controllo in 15 Paesi dell'UE, fra cui I'Italia, diversa normativa si ritrova nei Paesi Bassi e in Inghilterra dove il Khat può essere acquistato in negozi di ortofrutticoli e in minimarket (tra immigrati africani, soprattutto Somali, consumare il khat che è una pratica con una forte valenza culturale) ed in Svezia dove non è regolamentata.

Nello Yemen è il consumo di alcol è illegale e sanzionato mentre il consumo di Khat è libero; si calcolano come consumatori circa il $80 \%$ degli uomini e nel $45 \%$ delle donne.

In questo paese il consumo di Khat (si stima di circa il 16\% quota bilancio familiare) è un comportamento sociale condiviso, fortemente radicato e mirato a procurare euforia allontanando le sensazioni di fatica e fame aumentandole capacità comunicative all'interno del gruppo.

La pianta è ormai divenuta il secondo maggior prodotto di export in Etiopia, con proventi che superano il 15\% dell'export totale.

Kratom (Mitragyna speciosa) è un albero che cresce nel sud est asiatico ed in particolare in Tailandia ed è della stessa famiglia delle piante del caffè.

La pianta ha un tradizionale impiego come medicinale e come stupefacente, contiene numerosi alcaloidi del quale in più importante è la mitraginina.

Si utilizzano le foglie, solitamente polverizzate, oppure fatte bollire ricavandone una "pasta".
Usato nella medicina popolare come stimolante (a piccole dosi), sedativo (a dosi elevate), antidolorifico, medicinale contro la diarrea; un uso tradizionalmente fatto in Tailandia è come rimedio per la dipendenza da oppiacei.

Il meccanismo neurobiologico ancora non ben conosciuto, I'utilizzo cronico causa dipendenza con sintomatologia caratterizzata da spasmi muscolari, irritabilità, diarrea.

L'effetto compare solitamente 30-40 minuti dopo l'ingestione delle foglie e può durare un tempo significativo (5-6 ore).

Normativa: considerata l'elevata capacità di generare dipendenza il Kratom è stato vietato dalla Thailandia dal 1940 e dalla Malesia nel 2003.

Attualmente I'utilizzo è stato segnalato in modo significativo in Gran Bretagna, Svezia (dove sono stati anche segnalati casi di decesso) e negli Stati Uniti.

La Drug Enforcement Administration (DEA) ha inserito il Kratom nella lista delle sostanze psicoattive cui prestare attenzione. In Italia, come in molti paesi europei, il Kratom e I'alcaloide mitraginina non sono inclusi nelle tabelle della 309/90 e pertanto I'utilizzo è attualmente legale.

Sodio oxibato (acido $\gamma$-idrossibutirrico - $G H B$ ): il sodio oxibato fu inizialmente isolato e studiato nel 1960 da Henry Laborit, è stato usato come anestetico e ipnotico nel trattamento dell'insonnia. Sostanza diventata popolare negli anni ' 80 per il suo utilizzo come anabolizzante nelle palestre. Attualmente il GHB è usato, soprattutto in Italia, nel trattamento dell'etilismo.

Normativa: dal 1991 in Italia trova indicazione (con il nome commerciale di Alcover) nel trattamento dell'alcolismo, sia in acuto come antiastinenziale che nell'uso protratto per riduzione del craving.

In tale utilizzo clinico viene riscontata una buona efficacia trattamentale con scarsi episodi di misuso da parte dei pazienti, ovviabili comunque con un buon monitoraggio clinico.

Negli Stati Uniti d'America è stato per un certo tempo identificato in modo negativo con il nome di "liquid ecstasy" (enfatizzando un possibile uso come "rape drug") e dagli anni '90 è stato inserito nelle sulle sostanze controllate e dal marzo 2001 la Commissione sui narcotici ha posto il GHB nella tabelle delle sostanze illegali di cui è proibita l'importazione (non utilizzabile neanche per uso medico).

Fortuna che anche per questo farmaco "i tempi cambiano" e attualmente negli USA il sodio oxibato è in fase di sperimentazione per abuso alcolico e registrato per narcolessia (Xyrem).

Alcol: bevande alcoliche fermentate venivano già consumate nel neolitico, circa 9.000 anni fa nell'est asiatico.

Il vino appare chiaramente nei pittogrammi egizi già nel 4.000 a.C., mentre la birra era la principale bevanda alcolica dei Sumeri. Le civiltà precolombiane utilizzavano una bevanda alcolica ottenuta dalla fermentazione dell'agave. Dal 1.700 a.C. circa, la produzione e l'uso del vino dovevano diffondersi nella civiltà greca sino a diventare elementi centrali in molti rituali sacri.

Per quasi 10000 anni in occidente il consumo dell'acqua era poco frequente; la birra ed il vino, non l'acqua, sono state nel corso dei secoli le principali bevande, bevute quotidianamente da tutti e a tutte le età.

Il consumo di latte non era molto diffuso ed era considerato, in genere, un'abitudine "barbarica". Nel corso dei secoli l'acqua si era mostrata nociva e capace di provocare malattie acute e croniche, talvolta mortali. 
Di conseguenza si evitava di berla; anche perché spesso il suo sapore era sgradevole, diventando in poco tempo, se male conservata, putrida e maleodorante.

In Occidente non erano conosciuti metodi di depurazione e, a differenza di quanto avveniva in oriente, erano altresì ignoti $i$ vantaggi derivanti dalla bollitura dell'acqua.

Significativo notare che anche nella Bibbia non viene mai citata I'acqua come bevanda.

La quantità di alcol presente in queste bevande era di solito piuttosto bassa per cui anche gli effetti nocivi erano quasi del tutto inesistenti.

Nelle civiltà antiche, inoltre, I'alcol era considerato una fonte di calorie, e quindi di vero e proprio nutrimento.

La dieta di Milone di Crotone, uno dei più grandi lottatori di tutti i tempi, prevedeva otto chili di carne e cinque litri di vino al giorno.

Normativa: Platone (Dialoghi 395 a.C.) "Emaneremo innanzitutto una legge per cui i giovinetti, che ancora non abbiano compiuto i diciotto anni, non debbano assolutamente gustar vino, insegnando che non bisogna versare fuoco sul fuoco del corpo e dell'anima prima d'esser stati avviati a sopportar la fatica, per porre un freno alle esuberanti tendenze proprie della giovinezza..." Negli Stati Uniti dal 1919 al 1935 fu messa al bando fabbricazione, vendita, importazione e trasporto di alcool. (periodo definito "Proibizionismo" o "Il Nobile Esperimento").

La Legge islamica vieta rigorosamente le bevande alcoliche (oltre il gioco d'azzardo) perché "impediscono il normale funzionamento delle facoltà raziocinanti".
Questo è un precetto religioso, passato a normativa giuridica in molti paesi musulmani (dagli Stati del Golfo sino alla Malesia). In Iran le produzioni di alcol cessarono con la caduta dello Scia Reza Pahlavi. "In verità col vino e il gioco d'azzardo, Satana vuole seminare inimicizia e odio tra di voi e allontanarvi dal Ricordo di Allah e dall'orazione. Ve ne asterrete?" (Sacro Corano, Sura al-Ma'ida, 5:91) "Dio ha maledetto il vino, chi lo beve, chi lo serve, chi lo vende e lo compra, chi pesta le sue uva, chi lo trasporta e quello che lo ha ordinato e chi ne incassa il prezzo". In Europa sostanza di libera vendita con alcune limitazioni (ai minori, durante specifiche manifestazioni, ecc.).

\section{Sostanze psicotrope: quanto fanno male? Come possiamo misurarlo?}

Quindi è presumibile che una sostanza psicoattiva illegale si differenzi da quelle legali in quanto in grado di cagionare danni più gravi o comunque di un maggior rischio sulla salute individuale rispetto a i chi la consuma o globalmente "della società"?

Ci sono evidenze scientifiche su tali correlazioni?

Riguardo a queste domande riporto due scale, quella più datata del NIDA e quella più recente, pubblicata sul Lancet nel 2007. In quest'ultima tabella possiamo vedere che vengono analizzati più fattori inquadrabili nella possibilità di:

a) cagionare danno fisico,

b) creare dipendenza,

c) causare danno sociale.

National Institute of Drug Abuse (NIDA) - Scala di potenza di sostanze psicotrope (1 massimo, 6 minimo)

\begin{tabular}{|c|c|c|c|c|c|c|}
\hline & Astinenza & Rinforzo & Tolleranza & Dipendenza & $\begin{array}{c}\text { Intossicazione } \\
\text { acuta }\end{array}$ & Totale \\
\hline Eroina & 2 & 2 & 1 & 2 & 2 & 9 \\
\hline Alcol & 1 & 3 & 3 & 4 & 1 & 12 \\
\hline Nicotina & 3 & 4 & 2 & 1 & 5 & 15 \\
\hline Marijuana (the) & 6 & 5 & 6 & 6 & 4 & 27 \\
\hline Caffeina & 5 & 6 & 5 & 5 & 6 & 27 \\
\hline
\end{tabular}

Development of a rational scale to assess the harm of drugs of potential misuse

\begin{tabular}{|c|c|c|c|c|c|c|c|c|c|c|c|c|}
\hline & Danno & ico & & & Dipen & & & & Danno & sociale & & \\
\hline & Media & $\begin{array}{l}\text { Assunzione } \\
\text { acuta }\end{array}$ & $\begin{array}{l}\text { Assunzione } \\
\text { cronica }\end{array}$ & $\begin{array}{l}\text { Assunzione } \\
\text { endowenosa }\end{array}$ & Media & Piacere & $\begin{array}{l}\text { Dipendenza } \\
\text { psicologica }\end{array}$ & $\begin{array}{l}\text { Dipendenza } \\
\text { fisica }\end{array}$ & Media & Intossicazione & $\begin{array}{l}\text { Danno } \\
\text { sociale }\end{array}$ & $\begin{array}{l}\text { Costi per } \\
\text { la Sanith }\end{array}$ \\
\hline Eroina & 2.78 & 2.8 & 2.5 & 3.0 & 3.00 & 3.0 & 3.0 & 3.0 & 2.54 & 1.6 & 3.0 & 3.0 \\
\hline Cocaina & 2.33 & 20 & 2.0 & 3.0 & 2.39 & 30 & 2.8 & $1 \cdot 3$ & $2 \cdot 17$ & 1.8 & 2.5 & $2 \cdot 3$ \\
\hline Barbiturici & 2.23 & $2 \cdot 3$ & 1.9 & 2.5 & 2.01 & 2.0 & 2.2 & 1.8 & 2.00 & $2 \cdot 4$ & 1.9 & 1.7 \\
\hline Metadone & 1.86 & 2.5 & 17 & 1.4 & 2.08 & 18 & $2 \cdot 3$ & $2 \cdot 3$ & 187 & 1.6 & 1.9 & $2 \cdot 0$ \\
\hline Alcol & 1.40 & 1.9 & $2 \cdot 4$ & NA & 1.93 & $2 \cdot 3$ & 1.9 & 1.6 & $2-21$ & 2.2 & $2-4$ & $2-1$ \\
\hline Chetamina & 2.00 & 2.1 & 1.7 & $2 \cdot 1$ & 1.54 & 1.9 & 1.7 & 1.0 & 1.69 & $2 \cdot 0$ & 1.5 & 1.5 \\
\hline Benzodiazepine & 1.63 & 1.5 & 1.7 & 1.8 & 1.83 & 1.7 & 2.1 & 1.8 & 1.65 & 2.0 & 1.5 & 1.5 \\
\hline Anfetamine & 1.81 & 1.3 & 1.8 & $2 \cdot 4$ & 1.67 & 20 & 1.9 & 1.1 & 1.50 & 1.4 & 1.5 & 1.6 \\
\hline Tabacco & 1.24 & 0.9 & 2.9 & 0 & 2.21 & $2 \cdot 3$ & 2.6 & 1.8 & 1.42 & 0.8 & 111 & $2 \cdot 4$ \\
\hline Buprenorfine & 1.60 & 1.2 & $1 \cdot 3$ & $2 \cdot 3$ & 1.64 & 2.0 & 1.5 & 1.5 & 1.49 & 1.6 & 1.5 & $1 \cdot 4$ \\
\hline Cannabis & 0.99 & 0.9 & 2.1 & 0 & 1.51 & 1.9 & 1.7 & 0.8 & 1.50 & 1.7 & $1 \cdot 3$ & 1.5 \\
\hline Solventi & 1.28 & $2 \cdot 1$ & $1 \cdot 7$ & 0 & 1.01 & $1 \cdot 7$ & $1 \cdot 2$ & $0-1$ & $1-52$ & 1.9 & 1.5 & $1 \cdot 2$ \\
\hline 4-MTA & 1.44 & 2.2 & 2.1 & 0 & 1.30 & 1.0 & 1.7 & 0.8 & 1.06 & 1.2 & 1.0 & 10 \\
\hline LSD & 1.13 & 1.7 & 1.4 & $0-3$ & 1.23 & $2 \cdot 2$ & 1.1 & $0-3$ & 1.32 & 1.6 & $1-3$ & $1-1$ \\
\hline Metilfenidato & 1.32 & 1.2 & $1 \cdot 3$ & 1.6 & 1.25 & 1.4 & $1 \cdot 3$ & 1.0 & 0.97 & 1.1 & 0.8 & 11 \\
\hline Steroidi & 1.45 & 0.8 & 2.0 & 1.7 & 0.88 & 1.1 & 0.8 & 0.8 & 1.13 & 1.3 & $0-8$ & $1 \cdot 3$ \\
\hline$\gamma$-Idrossibutirrato & 0.86 & 1.4 & 1.2 & 0 & 1.19 & 1.4 & 1.1 & 1.1 & 1.30 & 1.4 & $1 \cdot 3$ & 1.2 \\
\hline Ecstasy (MOMA) & 1.05 & 1.6 & 1.6 & 0 & 1.13 & 1.5 & 1.2 & 0.7 & 1.09 & 1.2 & 1.0 & 1.1 \\
\hline Qat (Catha edulis) & 0.93 & 1.6 & 0.9 & 0.3 & 0.87 & 16 & 0.7 & 0.3 & 0.97 & 0.8 & 0.7 & 1.4 \\
\hline Arile nitrita & 0.50 & 0.3 & 1.2 & 0 & 1.04 & 16 & 1.2 & 0.3 & 0.85 & 0.7 & 1.1 & 0.8 \\
\hline
\end{tabular}

(David Nutt, Colin Blakemore, The Lancet, vol. 369, pp. 1047-1053, 24 March 2007) 
Leggendo queste tabelle (in cui le varie sostanze psicotrope sono elencate in ordine di danno decrescente) possiamo vedere come in essa sono elencate sostanze che, nella realtà italiana, sono illegali (droghe), sostanze legali e farmaci.

Prendendo come riferimento la più recente (Lancet) vediamo posizionarsi nei primi 14 posti:

- al $1^{\circ} 2^{\circ}, 8^{\circ}, 11^{\circ} 13^{\circ}$ e $14^{\circ}$ sostanze classificate dalla normativa (309/90) stupefacenti illegali;

- al $3^{\circ} 4^{\circ}, 6^{\circ} 7^{\circ} 10^{\circ}$ sostanze registrate come farmaci (alcuni di questi considerati illegali se utilizzati al di fuori di corretta prescrizione medica);

- al $5^{\circ}$ e $9^{\circ}$ sostanze legali voluttuarie;

- al $12^{\circ}$ sostanze impropriamente utilizzate come psicotrope (solventi).

Sembra pertanto che le scelte operate dai legislatori, in relazione alle normative che regolamentano la produzione, la vendita o la cessione di specifiche sostanze psicotrope, sia in un rapporto non diretto di causa-effetto con gli aspetti di salute pubblica o individuale.

Pare di vedere nell'impianto legislativo su tali sostanze delle valutazioni di più "ampio respiro"; assunte probabilmente non tanto in riferimento a valutazioni scientifiche relative alla specifica tossicità di quella data sostanza ma anche e piuttosto in relazione a molteplici altri fattori.

Tali fattori possono essere inquadrati in aspetti culturali propri di quella popolazione, aspetti religiosi, aspetti economici ma anche aspetti ideologici e/o etico morali.

Fattori questi ultimi che non di rado subiscono modifiche, nel percepito collettivo (il "sentire comune"), questo condiziona il variare della norma giuridica in chiave talvolta di maggiore tolleranza o altre volte di maggiore proibizione.

\section{E per concludere...}

Infine per rispondere ad una domanda rimasta in sospeso: recentemente I'American Psychiatric Association ha ufficialmente riconosciuto il "Caffeine Use Disorder" come vero e proprio problema di salute.

È quindi ipotizzabile un possibile inserimento della caffeina nei disturbi da uso di sostanze in un futuro DSM 6 ?

\section{Bibliografia}

Alter Ego, Droga e Cervello, Edizioni del Centro per la Diffusione della Cultura Scientifica, Università degli Studi di Cassino, 1995.

National Geographic, ERBA droga e medicina: la scienza della marijuana.

Porella B. (1994), Breve storia della cannabis (www.fuoriluogo.it/ eodp/storia.htm).

European Drug Report 2015, European Monitoring Centre for Drugs and Drug Addiction.

Nutt D., Blakemore C. (2007), The Lancet, vol. 369, 24 March: 10471053.

http://www.radicali.it/primopiano/20140122/legislazione-sullamarijuana-cannabis-terapeutica-nel-mondo.

https://it.wikipedia.org/wiki/Alimentazione_medievale.

https://it.wikipedia.org/wiki/Legalit\%C3\%A0_della_cannabis.

http://www.insostanza.it, Unità Operativa Prevenzione Dipendenze patologiche AUSL7 Siena.

http://dctf.uniroma1.it/galenotech/allucin.htm, Università di Roma.

https://drugs-forum.com/studies

http://www.druglawreform.info/en/publications/item/5184-infographichow-will-uruguays-regulation-of-cannabis-work.

http://www.emcdda.europa.eu/publications/drug-policy-profiles.

http://inchieste.repubblica.it/it/repubblica/rep-it/2013/12/05/ news/qat_l_oro_verde_dell_africa-72739026/.

http://www.repubblica.it/salute/2015/11/24/news/l_uso_di_ marijuana_in_italia_e_nel_mondo_ecco_i_dati-128051478/.

https://ballotpedia.org/California_Proposition_64,_Marijuana_ Legalization_\%282016\%29.

\section{RECENSIONE}

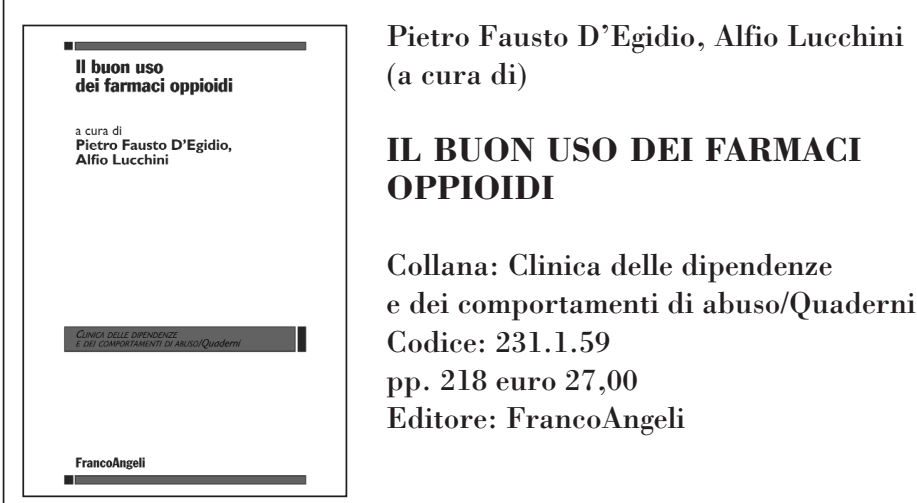

I farmaci oppioidi sono i presidi più significativi a disposizione dei clinici nei Servizi delle Dipendenze per curare le persone con dipendenza da eroina e oppiacei in genere.

La conoscenza dei meccanismi d'azione di questi farmaci è fondamentale, così come il loro uso ai dosaggi appropriati e l'attenzione a un'attiva relazione terapeutica.

I fenomeni del "misuso" - l'uso del farmaco per una via di somministrazione diversa dalla prescrizione medica - e della "diversione"
- la cessione o vendita del farmaco - sono ampiamente affrontati dalla comunità scientifica.

In questo volume sono raccolti i contributi presentati per l'XI premio nazionale FeDerSerD da professionisti del sistema d'intervento italiano sul tema della sicurezza d'uso, in particolare per i farmaci agonisti. Si tratta di diciotto lavori scientifici di oltre cento professionisti che testimoniano l'alto livello d'intervento e l'attenzione nel rapporto con il paziente che i Servizi offrono in Italia.

Il volume vuole coniugare le evidenze scientifiche con la ricerca applicata e la pratica clinica, nell'ottica di migliorare metodi e qualità del percorso con il paziente: un esempio che spinge alla fiducia anche in un momento tanto complesso per la tutela della salute nelle fasce deboli del Paese.

Pietro Fausto D'Egidio, medico, specialista in medicina interna, ematologia generale, allergologia e immunologia clinica, direttore del Servizio delle Dipendenze della ASL di Pescara, presidente nazionale di FeDerSerD.

Alfio Lucchini, medico, psichiatra, specialista in psicologia medica, psicoterapeuta, direttore del Dipartimento delle Dipendenze della ASST Melegnano e della Martesana, professore a contratto presso l'Università Cattolica di Milano, past president di FeDerSerD. 